\title{
A Novel Adaptive Compression Technique for Dealing with Corrupt Bands and High Levels of Band Correlations in Hyperspectral Images Based on Binary Hybrid GA-PSO for Big Data Compression
}

\author{
S. Kargozar Nahavandy \\ University of Tehran, Iran.
}

\author{
P. Ghamisi \\ University of Iceland, \\ Reykjavik, Iceland \\ M. S. Couceiro \\ Inst. of Syst. and Robotics \\ Ingeniarius, Lda \\ 3030-290 Coimbra, Portugal
}

\author{
L. Kumar \\ Ecosystem Management, Universi- \\ ty of New England, Australia
}

\begin{abstract}
Hyperspectral sensors generate useful information about climate and the earth's surface in numerous contiguous narrow spectral bands, being widely used in resource management, agriculture, environmental monitoring, among others. The compression of hyperspectral data helps in long-term storage and transmission systems. This paper introduces a new adaptive compression method for hyperspectral data. The method is based on separating the bands with different specifications by the histogram analysis and Binary Hybrid Genetic Algorithm-Particle Swarm Optimization (BHGAPSO). The new proposed method improves the compression ratio of the bestknown JPEG standards, saves storage space, and speeds up the transmission system. The proposed method is applied on two different test cases, and the results are evaluated and compared with a few powerful compression techniques, such as lossless JPEG and JPEG2000. The results confirm that the proposed method is accurate, simple and fast, which can be useful for big data (i.e, a high volume of data) processing.
\end{abstract}

\section{General Terms}

Algorithms.

\section{Keywords}

Remote sensing, Hyperspectral images, Image compression, Transformation, Binary Hybrid GA-PSO.

\section{INTRODUCTION}

Hyperspectral images have been widely used in numerous applications, such as resource management, agriculture, environmental monitoring, mineral exploration, and climate observation. This type of sensors can generate more than one terabyte data in one day. Because of these enormous data volumes, the use of a robust data compression technique has become very important for archiving and transferring purposes [1]. Due to the importance of generating highly accurate information about the atmosphere, clouds, and surface parameters provided by the hyperspectral sensors, lossy compression techniques are not acceptable in this case [2]. The economics of transmission and mass-storage of the large volumes of data accumulated by these sensors demonstrate that efficient compression is very important in this technology [3]. There are a few well-known methods for lossless compression, such as JPEG standards. These methods are generated for use on all kinds of images. In addition, there are some methods that are specifically designed for hyperspectral data. As hyperspectral data are composed of different bands that could have different specifications, applying a single method for all the bands without considering their specifications may not be an efficient means of compression.

In this study, a new adaptive compression method is introduced for hyperspectral data, which is composed of three different approaches. To this context, first, the corrupted bands are separated from the other bands and coded in a very efficient manner. Furthermore, the other bands are optimized by Binary Hybrid GA-PSO (BHGAPSO) and split into two groups based on their correlation. Then, each group is compressed with a new compression method. The proposed lossless method is based on Enhanced Differential Pulse Code Modulation Transformation (EDT) and suitable Huffman encoding. With respect to the experiments, the adaptive method leads to higher compression ratios than other investigated methods.

This paper is organized as follows. In Section 2, a few optimization algorithms are described. In Section 3, the proposed method, its structure, and specifications are presented. Section 4 is devoted to the experimental results and Section 5 presents the concluding remarks and possible future works.

\section{CURRENT OPTIMIZATION TECH- NIQUES}

\subsection{Particle Swarm Optimization (PSO)}

PSO is a very promising evolutionary computation technique in terms of simplicity and velocity [8]. PSO consists of a set of solutions denoted as swarm or population. Each solution consists of a set of parameters and represents a point in the multidimensional space, denoted as a particle [20]. Particles move through the search space with a specified velocity for finding the optimal solution. Each particle keeps a short-term memory, which helps it keeping track of its previous best position. Particles' positions are distinguished as personal, or local, best and global best. Particles' velocities are adjusted according to the historical behavior of each particle and its neighbors while flying through the search space. A particle's motion is highly affected by its current position and its memory of previous useful parameters, as well as the cooperation and knowledge of the swarm [8]. Therefore, the particles have a tendency to fly towards a better search area over the search process course [20]. The velocity of the $i$-th particle in the $k$-th iteration is determined as follows: 
$V_{i d}^{k+1}=W V_{i d}^{k}+c_{1} r_{1}\left(p b_{i d}^{k}-x_{i d}^{k}\right)+c_{2} r_{2}\left(g b_{d}^{k}-\right.$

$$
\text { xidk }
$$

where $c_{1}$ and $c_{2}$ are acceleration constants and $r_{1}$ and $r_{2}$ are random values in the range of 0 and 1 . The parameter $W$ is regarded as the inertia weight. The parameter $x_{i d}^{k}$ shows the position of each particle in the $d$-dimensional search space. The best previous position of each particle is represented by $p b_{i d}^{k}$ and called as particle best position. $g b_{d}^{k}$ is the best position of all particles, being denoted as global best particle. The $i$-th particle position is updated by:

$x_{i d}^{k+1}=x_{i d}^{k}+V_{i d}^{k}$

Kennedy and Eberhart [8] applied the sigmoid transformation on the velocity component to develop binary discrete PSO to control the range of velocity between 0 and 1 .

$\operatorname{Sigmoid}\left(V_{i d}^{k}\right)=\frac{1}{1+e^{-v_{i d}^{k}}}$

For updating the position of each particle:

$x_{i d}^{k}=\left\{\begin{array}{c}1, \text { if rand }<\operatorname{sigmoid}\left(V_{i d}^{k}\right) \\ 0, \text { otherwise }\end{array}\right.$

\subsection{Genetic Algorithm (GA)}

Genetic algorithm (GA) is an evolutionary optimization method based on the genetic process of biological organisms [7]. In comparison to other techniques, GA can emphasize much stronger on global, as opposed to local search and optimization [8]. Furthermore, GA is able to find an optimal solution without having to explore the whole (often vast) search space. GA starts optimization with several solutions. Each of these solutions is called chromosome or individual, consisting of several genes which can have different values. These genes carry the attributes of each individual, representing the fitness value of the chromosome. A set of the chromosomes constitute a population, in which the most fitted ones are selected for generation. For the generation phase, the two most fitted chromosomes are selected and their chromosomes are combined to make a new offspring (or solution). The act of combination is done by crossover, followed by a mutation mechanism applied on each child individually [7]. As the PSO algorithm, this cycle is iteratively repeated until a termination criterion is met [9].

\subsection{Hybrid GA-PSO (HGAPSO)}

The hybrid algorithm combines the standard velocity and updates rules of PSOs with the ideas of selection, crossover and mutation from GAs. In [22] and [23], Ghamisi et al. proposed the HGAPSO for supervised feature selection and segmentation. In this work, the concept of HGAPSO is further developed in order to handle unsupervised feature selection and, finally, data compression.

The PSO algorithm presents several drawbacks, from which one may highlight its premature convergence. The underlying principle behind this problem is that particles converge to a single point between the global and the local best positions. This point is not guaranteed to be optimal [18]. Additionally, the fast rate of information flow between particles results in similar particles with a loss in diversity, thus increasing the possibility of being trapped in local optima.

Another drawback is that stochastic approaches have problemdependent performance. This dependency is related with the set of parameters of the algorithm. A different parameter settings for a stochastic search algorithm results in high performance variances [5]. Moreover, each problem may have an "optimal" set of parameters. As an example, increasing the inertia weight will increase particles' speed, resulting in more exploration (global search) and less exploitation (local search), and vice-versa. Hence, finding the best value for a given parameter is not an easy task and may differ from one problem to another [11].

Despite these drawbacks, the PSO algorithm is conceptually simple and can be implemented in a few lines of code. As previously stated, the PSO also have memory, whereas in a $\mathrm{GA}$, if an individual is not selected, the information contained by that individual is lost. However, without a selection operator, PSOs may waste resources on poor individuals. A swarm interaction enhances the search for an optimal solution, whereas GAs have trouble finding an exact solution and are best at reaching a global region [12]. Hybrid GA models are often used to overcome this problem. The authors in [19], it is said that the correct combination of both models has the potential to achieve better results faster and to work well across a wide range of problems. Both Angeline [13] and Eberhart [12] have suggested that a hybrid combination between GA and PSO could produce a very effective search strategy

The Hybrid GA-PSO is made for optimization of problems in continuous and multidimensional search spaces [13]. To perform the feature selection, the Hybrid GA-PSO needs to be extended in order to deal with binary data.

\section{EXTENDED NEW LOSSLESS COM- PRESSION METHOD (ENLCM)}

In this section, a novel adaptive compression method is proposed. This new method is based on NLCM. For further information regarding the NLCM, please see [16], [17] and [24].

Figure 1 illustrates the general engine of the proposed method, based on NLCM. First, the histogram of input hyperspectral data is analysed. Subsequently, corrupted bands are separated from other bands. Although these bands do not carry information, they should also be saved since they are required in the reconstruction phase for placing the bands in the correct orders. When the corrupted bands are separated, they are coded in the smallest form possible. Ten bytes are considered for this purpose, which will be described afterwards. The other bands include the most important information. However, hyperspectral imaging systems are able to acquire several hundreds of adjacent spectral information, from the visible to the infrared region, in which neighbouring spectral bands are usually highly redundant [4]. After separating corrupted from uncorrupted bands, the group of bands with the lowest correlation with each other (i.e., the bands that contain more information) are selected. These bands are compressed with the proposed lossless scheme method. Furthermore, the remaining bands are compressed by the proposed visually lossless method. Finally, the outputs of these three compression steps are added together to create the final compressed bit-stream, which is ready for storage or transmission. The compressed bit-stream is the coded version of the input hyperspectral data. 




Fig. 1: General Engine of ENLCM

1) Compression of Corrupted Bands: The first step of the proposed method is the histogram evaluation, where the histogram of each band is analysed to determine whether it is corrupted or not. In this method, any band that has only one value for its histogram is a corrupted band. In brief, an uncorrupted band has different intensity values, while a corrupted band has only one value, which is usually 0 or 255 . In this paper, only the bands with one value are compressed by this coding scheme. When the histogram is evaluated and the corrupted bands are determined, the coding step begins. In the coding step, 10 bytes are assigned for the corrupted bands, where specific codes are assigned for the starting and finishing 2 bytes. These code words separate them from the other bands and help in the reconstruction phase. The third byte indicates the number of corrupted band, the fourth byte is used to show the intensity value of the band, and the other bytes are aligned for the unpredictable headers. Figure 2 illustrates these bytes.

\begin{tabular}{|c|c|c|c|c|}
\hline 2 & 3 & 4 & $\ldots$ & 10 \\
\hline Start Code & Band \# & Intensity & Considered Header & Finish Code \\
\hline
\end{tabular}

\section{Fig. 2: Contents of 10 assigned bytes for corrupted bands}

2) Compression of Uncorrupted Bands: As can be seen in Figure 1, at first, a group of bands with the lowest correlations with each other is selected. In this paper, in order to find the bands with the lowest correlation, Hybrid GA-PSO was used. The basic Hybrid GA-PSO algorithm is made for optimization of problems in continuous, multidimensional search spaces [23]. To perform the feature selection and select the uncorrelated bands among the rest, the conception of the Hybrid GAPSO is needed to be extended in order to deal with binary data [22].

\subsection{Binary Hybrid GA-PSO (BHGAPSO)}

In this paper, we develop a new binary hybrid GA-PSO (BHGAPSO) method, which was first proposed in [22]. This optimization approach is effective for the optimization of hyperspectral bands by considering the data specification. As shown in [5], although the performance of this optimization approach is similar to the GA algorithm, it is able to achieve the same result in a lower number of iterations.

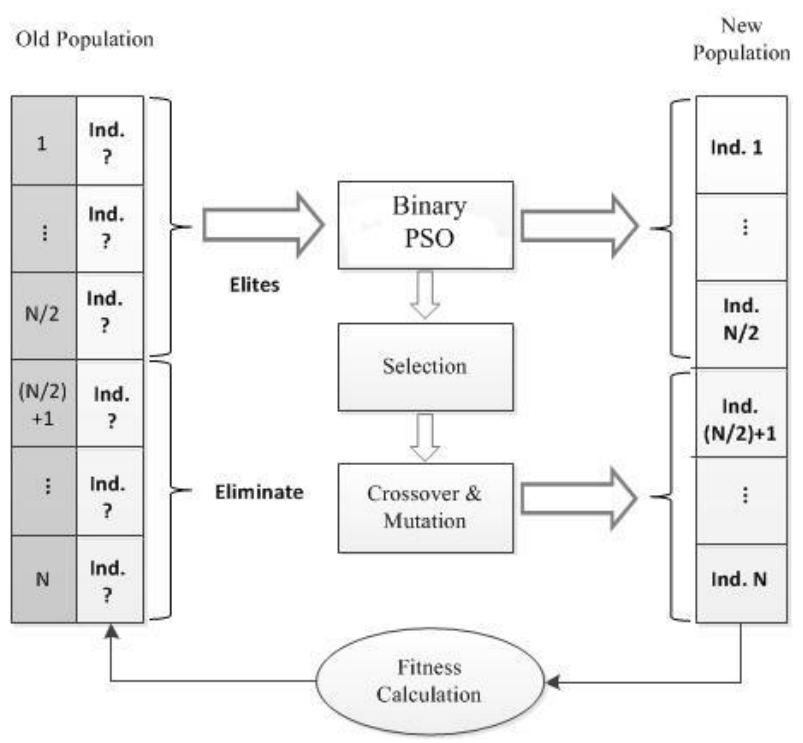

Fig 3: General engine of the BHGAPSO

Figure 3 shows the block diagram of the BHGAPSO. The total number of variables in each velocity and position component is equal to the total number of uncorrupted bands. Each position variable can be 0 or 1 , which shows the presence or absence of the specific band, respectively. The velocity is interpreted as a probability to change a bit from 0 to 1 , or from 1 to 0 , when updating particles' position. These individuals may be regarded as chromosomes in terms of GA, or as particles in terms of PSO. Afterwards, new individuals are created by enhancement, crossover and mutation operations. For clarity, the flow of these operations is illustrated in Figure 3 and described as follows:

Enhancement: In each generation, after the fitness values of all the individuals in the same population are calculated, the top-half best-performing ones are marked and identified as elites. Instead of reproducing the elites directly to the next generation as elite GAs do, we first enhance the elites using the Binary PSO (BPSO) algorithm. By using these enhanced elites as parents, the generated offsprings will usually achieve better performance than those bred by original elites. The elite group is regarded as a swarm and, as such, equations (1)-(4) are applied to the elites. In each iteration, the range of velocity is regulated in the range of 0 and 1 by sigmoid function and compared with the random chromosome between 0 and 1 and the position in the binary format is updated. By performing PSO on the elites, we increase the search ability of the algorithm. Half of the population in the next generation is occupied by the enhanced individuals, the remainder by crossover operation.

Crossover: To select parents for the crossover operation, the tournament-selection scheme is used, in which two enhanced elites for each parent are selected at random. The fitness values between pairs are compared and the two elites with a better fitness value are selected as parents. Two offsprings are created by performing crossover on the selected parents. Twopoint crossover operation is used, where two crossover sites are randomly selected within the range of an individual and swapping occurs. These produced offsprings occupy half of the population in the next generation. 
Mutation: In BHGAPSO, mutation occurs in conjunction with the crossover operation. Here, uniform mutation is adopted, i.e., the mutated gene is drawn randomly, uniformly from the corresponding search interval. In this paper, a constant mutation-probability equal to 0.1 was considered [21].

After running the BHGAPSO, a solution with the best fitness function value is selected as the best particle. The output of this step is the fittest individual in the binary format. The length of this individual is equal to the total number of bands. Each gene is illustrated with the presence of the specific band. After decoding this solution, a group of bands with fewer correlations is determined. This group is compressed with our lossless compression method. The remaining bands are coded by the following proposed compression methods.

\subsection{Lossless Algorithm}

In this paper, for optimization of hyperspectral bands, correlation coefficient was selected as an index for calculating correlation values (corr) of the bands. The correlation coefficient is an index which can measure how much two variables change together. The range of the correlation coefficient is between -1 to 1 . If the absolute value of the correlation coefficient is around 0, it shows two bands have less correlation with each other and contain more variable information. The correlation coefficient is calculated as follow:

$\operatorname{cost}\left(X_{k}\right)=\sum_{i=1}^{n} \sum_{j=1}^{n}\left|\frac{\left.\operatorname{corr}\left(v(i)_{k}, v(j)_{k}\right)\right)}{N u m}\right|$

$v(j) \neq 0 \quad v(i) \neq 0 \quad i \neq j$

$\operatorname{corr}(v(i), v(j))=\frac{\operatorname{cov}(v(i), v(j))}{\sigma_{v(i)} \times \sigma_{v(j)}}$

where $v(i)_{k}$ and $v(j)_{k}$ are the $i$-th and $j$-th variables in the $k$ th particle. Num is the total times that correlation is calculated for each particle in each iteration. $\sigma_{i}$ and $\sigma_{j}$ are the standard deviations of the $j$-th and $i$-th variables, respectively.

The uncorrupted bands are optimized by BHGAPSO and a group of bands with the lowest correlation is found. This group of bands is compressed by the proposed lossless algorithm (or NLCM), which comprises the EDT and the optimized Huffman encoder, and works as follows:

1) EDT: This method was introduced in [24], which is an efficient transformation due to its high capability in terms of redundancy reduction and energy compaction. In addition, it depicts an improved prediction performance when compared with the previous predictive schemes, such as DPCM. As it can be seen in Figure 4, for transforming an image by EDT, the input image intensities are manipulated first. The new intensities are predicted by one of the prediction equations shown in Figure 5. After predicting the new image intensities, the predicted image is retrieved through manipulation once again, in which the prediction error can be easily calculated by subtracting the main image from the predicted image.

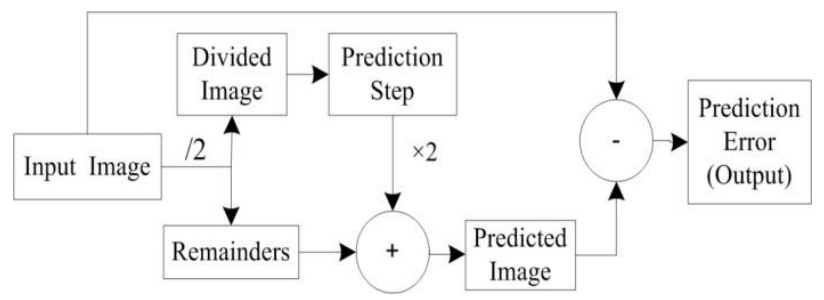

Fig. 4: Block diagram of EDT [17]



\begin{tabular}{l|l|l} 
1. $X=(B+D) / 2$ & 4. $X=D$ & 7. $X=B+(D-A) / 2$ \\
\hline 2. $X=B+D-A$ & 5. $X=B$ & 8. $X=(A+B+C+D) / 4$ \\
\hline 3. $X=D+(B-A) / 2$ & 6. $X=A$ & 9. $X=(B+C) / 4+D / 2$
\end{tabular}

Fig. 5: Neighboring Pixels and Prediction Equations [5]

This paper considers the entropy analysis to evaluate the EDT transformation. The entropy value is a measure for the possibility of compression by encoding. Entropy indicates the required bit per pixel amount. In fact, the entropy shows how much an image may be compressed in bits per pixel. For example, when one says that the entropy of an 8-bit image is 6.3, it means that the required bits per pixel amount is 6.3 for that image. Therefore, such range of compression is possible for that image. Also, according to Shannon's first theorem [10], it is possible to reach an entropy value using an encoder. The entropy value $(E)$ of any given image is calculated as follows:

$E=\sum_{k=0}^{L-1} p_{r}(r) \log _{2} p_{r}(r)$

where $r$ is the intensity value, $L$ is the number of intensity values used to present the image, and $p_{r}$ is the probability of intensities. For an image with a 8-bit depth, $L$ would be equal to 256 and $r$ would be in the range of $0-255$. The histogram of the given image can be used to calculate the intensities' probabilities [10]. A lower entropy value indicates that more compression is possible. Transformation increases energy compaction and, as a result, the probability of each intensity or source symbols increases, which leads to less redundancy and less entropy.

2) Optimized Huffman Encoding: Huffman codes the image intensities based on their probability and converts them as a bit-stream. Therefore, the Huffman dictionary, which includes intensity codes, needs to be saved as an overhead. After calculating the prediction error, all the values remain in the range of $-2^{k}+1$ and $2^{k}-1$. For example, for a grayscale image with an 8bit depth, this interval remains between -255 and 255 . Therefore, Huffman encoding may need a dictionary that could cover this interval. However, the transformed image by EDT has only even values. To have an optimized entropy encoder, the proposed method uses only even values of the aforementioned interval for the Huffman dictionary. As a result, a smaller dictionary is achieved and less header is needed.

3) Reconstruction: The reconstruction phase consists of Huffman decoding and inverse transform of our transformation method. The inverse of the transformation is obtained by calculating the main values from the inverse of prediction equation. To achieve this goal and to have a complete reconstruction, the first row and the first column of the main image need to be saved and then, reconstruct each pixel from neighbours. Inverse transformation for pixel X of Figure 5 (i.e, $(I, j))$ is calculated as follow:

$I(i, j)-I(i, j) \bmod n=$

$T(i, j)+n\left[\frac{\text { predictionbyequation } \# k}{n}\right\rfloor$

where $k$ is the equation number from Figure $5 . I(i, j) \operatorname{modn}$ is the reminder which was saved as the overhead. $T(j, j)$ is the predicted image.

\subsection{Visually-Lossless Algorithm}

For near-lossless or visually lossless transformation, $n$ is equal to 4 and reminders are eliminated. This causes at most $1.56 \%$ intensity change in the reconstruction of the transformed image. By increasing the value of $n$, the compression ratio for each band is increased. If a very large $n$ like 64 is 
picked, a lot of information may be lost and the predicted image does not have an acceptable quality and, as a result, the reconstructed image will not have a good quality, respectively. Therefore, it depends on the importance of the required quality for picking a divisor. The transformation depends on the needed quality and the environment in which the image is compressed, which is a functionality of the new method. For hyperspectral image compression, lossless or visually-lossless transformation is preferred. Signal to noise ratio (SNR) can be used for near lossless or visually lossless compression to see if the reconstructed image is acceptable or not. SNR of at least 5 is required to be able to distinguish image features at $100 \%$ certainty [14]. However, the lossless scheme does not need this kind of evaluation, and the compression efficiency is the only relevant factor [5]. Equation (9) is used for calculating the SNR [15]. To calculate the signal to noise ratio in decibels (dB), equation (10) is considered. An SNR is calculated between the original image and the reconstructed image to evaluate how similar these are.

$$
\begin{aligned}
& S N R=\frac{\sum_{i=0}^{M-1} \sum_{j=0}^{N-1}\left(R(i, j)^{2}\right)}{\sum_{i=0}^{M-1} \sum_{j=0}^{N-1}(I(i, j)-R(i, j))^{2}} \\
& S N R_{d b}=10 \log _{10} S N R
\end{aligned}
$$

\section{EXPERIMENTAL RESULTS}

In this work, two widely used hyperspectral data sets have been taken into account. The first test case was a hyperspectral image with 242 bands. Each band consists of $256 \times 3128$ pixels and was captured by Hyperion, i.e., one of the hyperspectral sensors that is carried on the Earth Observer (EO-1) satellite in a 705-km sun-synchronous orbit at a 98.7-degree inclination, providing a resolution of the surface properties in hundreds of spectral bands in the range of $0.4-2.35 \mu \mathrm{m}$ at $30 \times 30 \mathrm{~m}$ spatial resolution [6]. Figure 6 shows the 44th band of the first test case.

The second test case was captured by the Hyperspectral Digital Imagery Collection Experiment (HYDICE) sensor, which collected data in 210 bands in the range $0.4-2.5 \mu \mathrm{m}$ at an IFOV of 1-4 m, depending on the altitude of the aircraft and ground speed. In this case, the sensor collected data over the Washington DC Mall in 190 bands. This data set contains 1280 scan lines with 307 pixels in each scan line, i.e., each band is a $1280 \times 307$ image (Figure 6).



Fig. 6: A selected band for the first (top) and second (bottom) test cases.

Figure 7 illustrates the entropy value of these two hyperspectral data test cases. As can be seen, the entropy value of some bands in the first test case is equal to zero. This implies that they have only one value for all the pixels in that band, and one can consider them as the corrupted bands. It can be seen that, in the first test case, there are 62 corrupted bands, indicating that more than $25 \%$ of the bands are corrupted. In the second test case, it can be seen that there are just a few bands with an entropy equal to zero, as well as some others with low entropy. These are highly correlated in the neighbouring pixels and include less amount of information when compared with the other bands. The compression ratio of these bands is expected to be more than the others. In addition, the proposed methods include the predictive scheme and, due to the neighbouring correlation of the large amounts of bands, it can be efficient for this test case as well.

In the proposed Adaptive Method, the sum of the compressed bit-streams consists of the coded version of the corrupted bands and the compressed bit-streams obtained from the proposed visually-lossless and lossless compression methods.


Fig. 7: The Entropy Values of All the Bands of a) First Test Case, b) Second Test Case [24].

Figures 8 and 9 illustrate the variation of fitness function obtained by the GA and BHGAPSO methods with varying numbers of iterations. The results show that BHGAPSO is able to find a better solution in less number of iterations than a situation when the GA is taken into account.

The new method was applied on the hyperspectral data test cases, and the compression ratios are presented in Table 1. The compression ratio $(C R)$ was calculated as:

$C R=\frac{\text { Sum of All Bands Sizes }}{\text { Sum of Compressed Bit Stream Sizes }}$

Table 1. Compression Ratio for Compression of Both TestCases Obtained by Different Compression Algorithms

\begin{tabular}{|c|c|c|c|c|}
\hline Test case & $\begin{array}{c}\text { Lossless } \\
\text { Method } \\
\text { (NLCM) }\end{array}$ & $\begin{array}{c}\text { Adaptive } \\
\text { Proposed } \\
\text { Method } \\
\text { (ENLCM) }\end{array}$ & JPEG2000 & $\begin{array}{c}\text { Lossless } \\
\text { JPEG }\end{array}$ \\
\hline First & 2.58 & 3.76 & 2.778 & 2.327 \\
\hline Second & 3.75 & 4.08 & 2.04 & 2.375 \\
\hline
\end{tabular}

Figures10 and 11 illustrate the initial population, the population after eliminating the corrupted bands and the last population after optimizing hyperspectral bands by the BHGAPSO 
method. The population converges to some specific bands for both test cases. These bands have the highest correlation with each other and may provide more information than other bands.
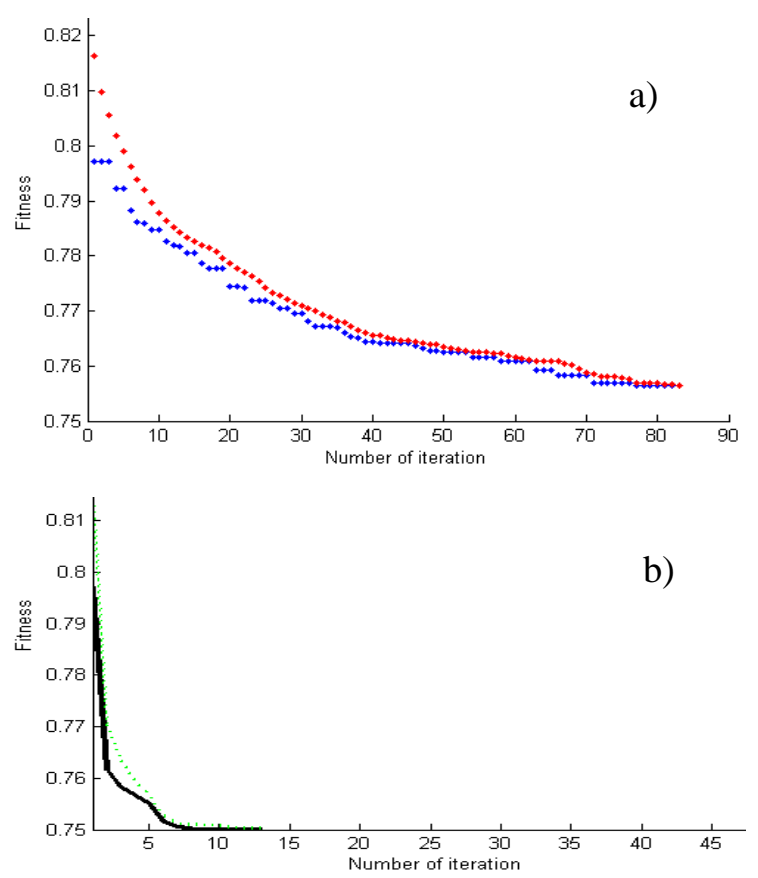

Fig. 8. Variation of Cost Function for different iterations in a) GA b) BHGAPSO for the first test case
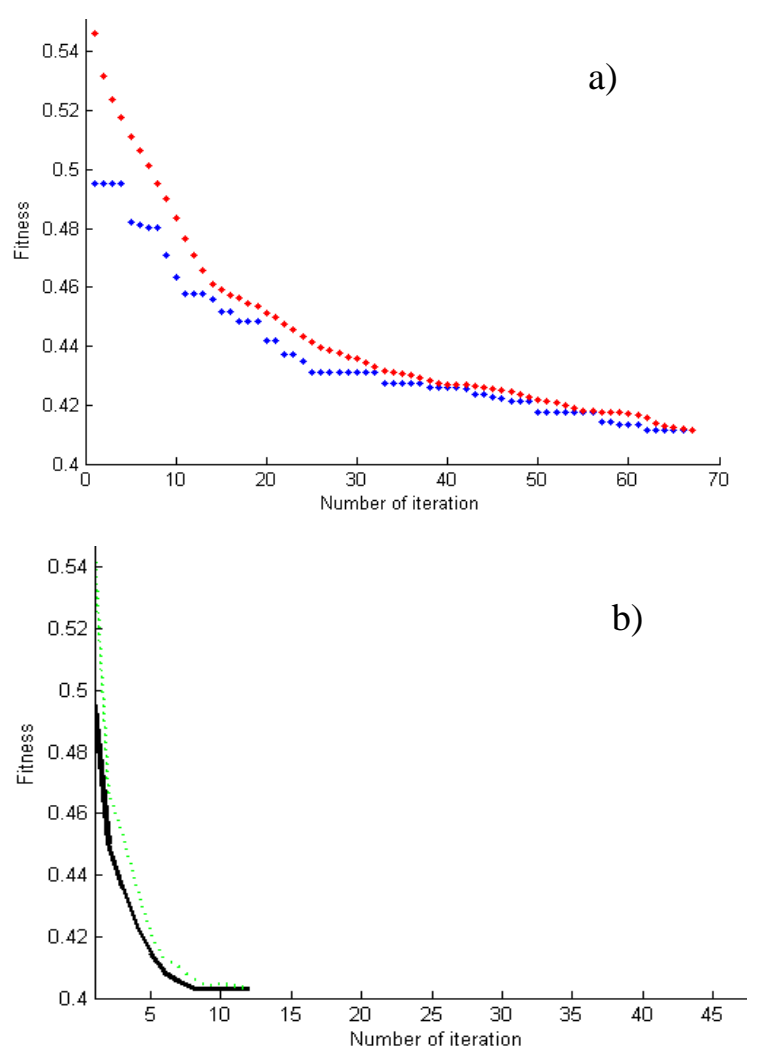

Fig. 9. Variation of Cost Function for different iterations in a) GA b) BHGAPSO for the second test case

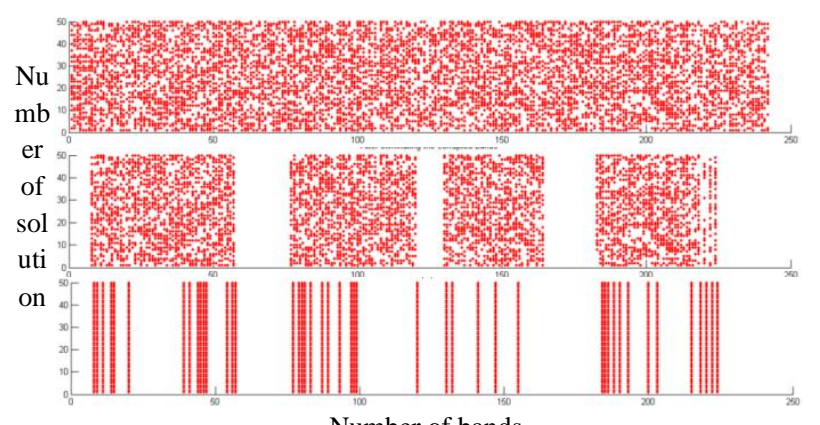

Number of bands

Fig 10: Initial popurauvu ( wop), un population after eliminating the corrupted bands (middle), last population after optimizing hyperspectral bands (bottom) by BHGAPSO for the first test case

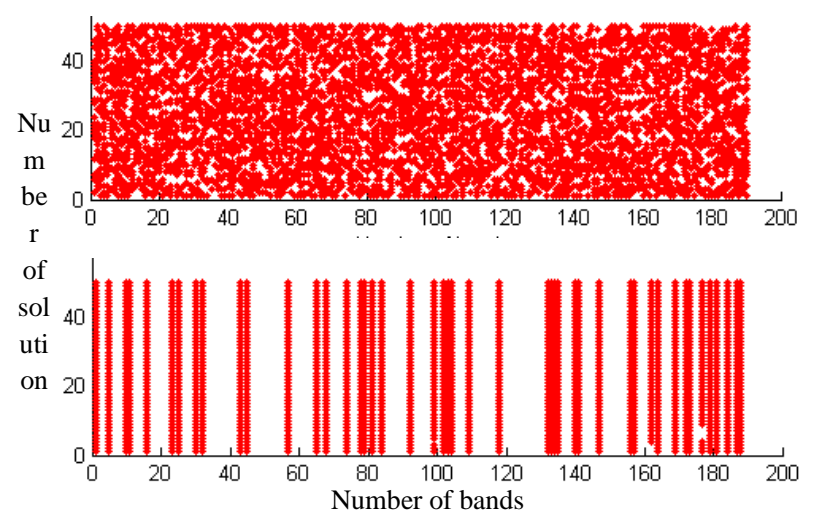

Fig 11: Initial popurauon (cop), rast population (bottom) after optimizing hyperspectral bands by BHGAPSO for the second test case

Figure 12 illustrates the input and output images after the reconstruction phase, and their histograms for band 112 for the first test case. As can be seen, the output image is visually lossless. The SNR values for some sample bands which were compressed by the visually lossless scheme of proposed method were calculated for both test cases and the results are presented in tables 2 and 3. The SNR evaluation is devoted to the visually lossless scheme where remainders are omitted to have more compression. As can be seen from Tables 2 and 3, SNR values are acceptable according to what has been discussed in the previous sections.

Figures 13 and 14 show the compression ratio of different methods for all the bands. According to Figure 13, in the first test case, the proposed adaptive method (ENLCM) gives the best result in terms of compression ratio, while the lossless method (NLCM) works better than the lossless JPEG. However, JPEG2000 has a better compression ratio than the NLCM because of better compression of the corrupted bands. The only advantage of NLCM is its simplicity, which is suitable for real-time systems that need a simple and fast algorithm. ENLCM works efficiently and compresses each band as required. Compared to the previous methods, ENLCM demonstrates an increased compression ratio of more than $15 \%$, while JPEG 2000 shows an increase of more than 5\%.

Table 2. SNR Values for the First Test Case

\begin{tabular}{|c|c|}
\hline Band \# & SNR \\
\hline $\mathbf{1 0}$ & 92.3553 \\
\hline $\mathbf{1 0 0}$ & 122.0417 \\
\hline $\mathbf{1 5 0}$ & 121.9455 \\
\hline
\end{tabular}




\begin{tabular}{|c|c|}
\hline Average & $\mathbf{1 2 0 . 5 2 7 4}$ \\
\hline
\end{tabular}

Table 3. SNR Values for the Second Test Case

\begin{tabular}{|c|c|}
\hline Band \# & SNR \\
\hline $\mathbf{2 0}$ & 109.5706 \\
\hline $\mathbf{1 0 0}$ & 85.1566 \\
\hline $\mathbf{1 5 0}$ & 76.3652 \\
\hline Average & $\mathbf{1 0 6 . 2 8 1 0}$ \\
\hline
\end{tabular}
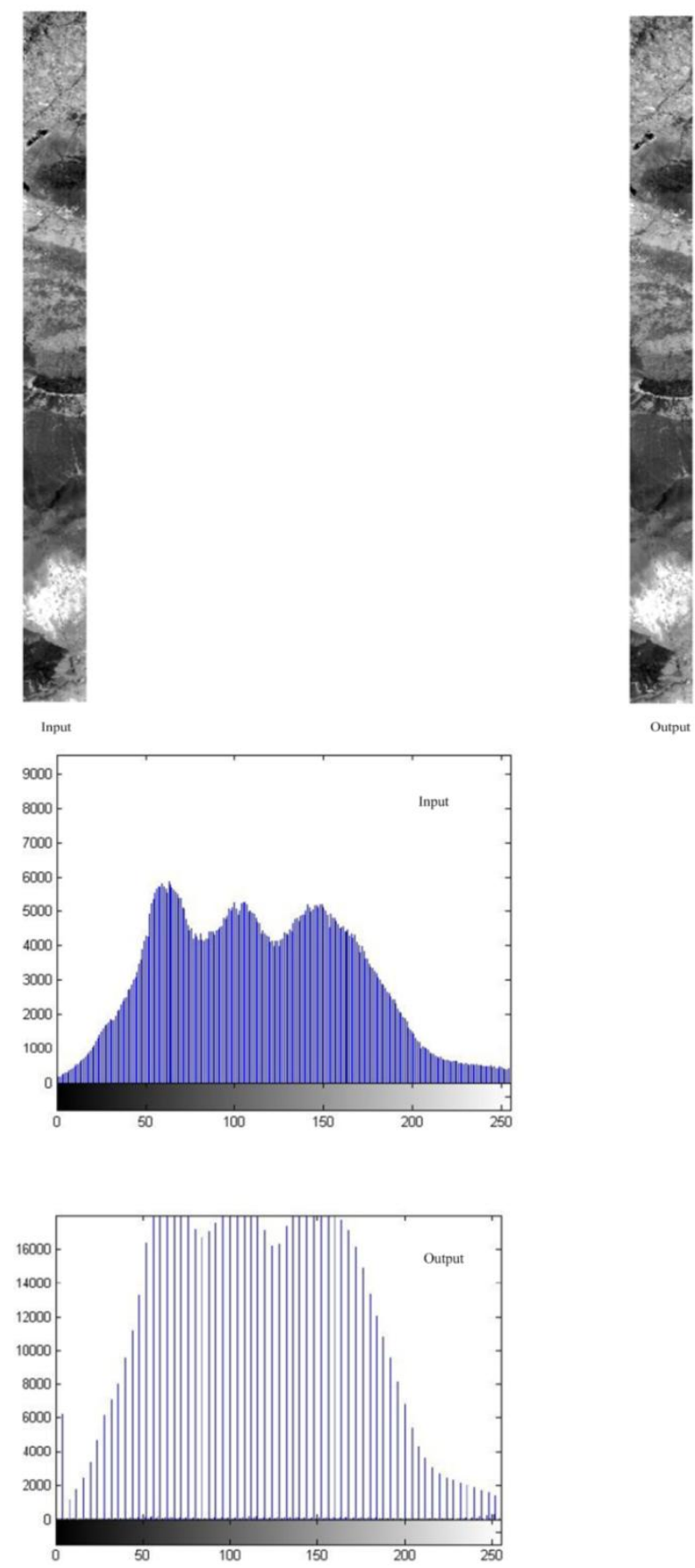

Fig. 12. The input image and output image after reconstruction phase, and their histograms for the first test case

As shown in Fig. 13, the ENLCM and NLCM methods work more efficiently for the compression of uncorrupted bands.
However, in the corrupted bands, the compression ratio of the proposed adaptive method is much higher than the others. Therefore, ENLCM works better than other compression techniques. It should be noted that, for visualization purposes, compression ratio with a value superior to 5 are not included in the figure because the values of the compression ratio of the corrupted bands are very high. For instance, the corrupted band number 75 has a compression ratio of 8.25 and 1190.19 for lossless JPEG and JPEG2000, respectively. However, the proposed adaptive method is able to achieve a compression ratio of 82599. In addition, it is clear from Fig. 13 that the proposed methods are better than the JPEG2000 for most of the bands.

ENLCM also demonstrates the best result in the second test case (Fig. 14). For the second test case, JPEG2000 shows the worst result among the three methods. It shows that a predictive scheme is better for this test case. Also, due to the improvement of the proposed method with respect to Lossless JPEG, one can conclude that the predictive scheme could be a good choice for hyperspectral data compression. As expected, the compression ratios are high in the low-detail bands with lower entropy. Furthermore, the efficiency of the proposed method is visually tangible. In the second test case, about $50 \%$ improvement in the compression ratio could be observed and, in this case, the compression system is computationally simple.



Fig. 13. The Compression Ratio of Different Methods for All the Bands of the First Test Case



Fig. 14. The Compression Ratio of Different Methods for All the Bands of the Second Test Case

\section{CONCLUSION}

In this study, due to the specification of the hyperspectral data, a novel method (ENLCM) for the compression of hyperspectral data has been introduced, which acts differently on each band depending on its specification. ENLCM is based on separating the corrupted bands from the others, optimizing the 
hyperspectral bands, and finding a group of bands with less correlation with each other. Corrupted bands are separated based on a histogram evaluation step and compressed using a new 10-bytes coding scheme. Afterwards, in order to find a group of bands with less correlation with each other, the uncorrupted bands are optimized using the BHGAPSO. The output of this step is a chromosome which contains a group of bands with the least correlation value. This group is compressed with our lossless compression method. The remaining bands are coded by the proposed visually lossless compression method. Finally, the outputs of these three compression steps are added together to create the final compressed bitstream, which is ready for storage or transmission. Experimental results show that ENLCM is much more efficient than NLCM, JPEG and JPEG2000, with an overall improved compressed ratio. ENLCM was tested on two different cases and compared with JPEG standards. In the first test case, more than $12 \%$ improvement in terms of the compression ratio is obtained compared to the other methods, and about 50\% improvement was observed in the second test case.

\section{REFERENCES}

[1] M. R. Pickering and M. J. Ryan, hyperspectral data compression, G. Motta, F. Rizzo, and J. A.Storer, Eds. Springer, 2006, chapter 1 .

[2] J.Mielikainen and P.Toivanen, hyperspectral data compression, G. Motta, F. Rizzo, and J. A.Storer, Eds. Springer, 2006, chapter 2 .

[3] S. R. Tate, "Band ordering in lossless compression of multispectral images," IEEE Transaction on Computers, vol. 46, no. 4, pp. 477-483, 1997.

[4] T. Ebrahimi, D. S. Cruz, J. Askelof, M. Larsson, and C. Christopoulos, "Jpeg 2000 still image coding versus other standards," in SPIE Int. Symposium, San Diego California USA, $30 \mathrm{Jul}$ - 4 Aug 2000, invited paper in Special Session on JPEG2000.

[5] P. Ghamisi, F. Sepehrband, J. Choupan, M. Mortazavi, "Binary Hybrid GA-PSO Based Algorithm for compression of Hyperspectral Data", The $5^{\text {th }}$ International Conference on Signal Processing and Communication Systems (ICSPCS' 11), 12-14 December, Honolulu, Hawaii.

[6] A. Skodras, C. Christopoulos, and T. Ebrahimi, "The JPEG2000 still image compression standard," IEEE Signal Processing Magazine, pp. 36-58, sept 2001.

[7] Goldberg, D. E. (1989). Genetic algorithms in search, optimization, and machine learning. Reading, Massachusetts, USA: Addison-Wesley Longman.

[8] J. Kennedy and R.C. Eberhart, Swarm Intelligence, Morgan Kaufmann Publishers, San Francisco, 2001.

[9] P. Ghamisi, M. S. Couceiro, J. A. Benediktsson, A Novel Feature Selection Approach Based on FODPSO and SVM, IEEE Transaction on Geoscience and Remote Sensing, [In press].

[10] P. Ghamisi, M. S. Couceiro, N. M. F. Ferreira, L. Kumar, "Use of Darwinian Particle Swarm Optimization technique for the segmentation of Remote Sensing images", IGARSS 2012, pp.4295-4298, 22-27 July 2012.

[11] K. Premalatha and A.M. Natarajan, Hybrid PSO and GA for Global Maximization, ICSRS Publication, Vol. 2, No. 4, December 2009
[12] Russell C. Eberhart and YuhuiShi.Comparison between genetic algorithms and particle swarm optimization. In et. al. V. William Porto, editor, EvolutionaryProgramming, volume 1447 of Lecture Notes in Computer Science, pages 611-616. Springer, 1998.

[13] Peter J. Angeline. Evolutionary optimization versus particle swarm optimization: Philosophy and performance differences. In V. William Porto and et al., editors, Evolutionary Programming, volume 1447 of Lecture Notes in Computer Science, pages 601-610. Springer, 1998.

[14] T. Bushberg, The essential of Medical Imaging, 2nd ed. Philadelphia: Lippincott Williams \& Wilkins, 2006.

[15] R. Gonzales and R. Woods, Digital Image Processing, 3rd ed. New Jersey: Pearson Prentice Hall, Upper Saddle River, 2008, pp. 525-626.

[16] P. Ghamisi, A. Mohammadzadeh, M. R. Sahebi, F. Sepehrband and J. Choupan, "A Novel Real Time Algorithm for Remote Sensing Lossless Data Compression based on Enhanced DPCM", International Journal of Computer Applications 27(1):47-53, August 2011.

[17] F. Sepehrband, P. Ghamisi, A. Mohammadzadeh, M. R. Sahebi, J. Choupan, "Efficient Adaptive Lossless Compression of Hyperspectral Data Using Enhanced DPCM", International Journal of Computer Applications 35(4):611, December 2011.

[18] F. Van den Bergh. An Analysis of Particle Swarm Optimizers, PhD Thesis. Department of Computer Science, University of Pretoria, South Africa, 2002.

[19] P. Ghamisi, "A Novel Method for Segmentation of Remote Sensing Images based on Hybrid GA-PSO", International Journal of Computer Applications 29(2):7-14, September 2011.

[20] P. Ghamisi, Micael S. Couceiro, Jon Atli Benediktsson, Nuno M. F. Ferreira "An Efficient Method for Segmentation of Images Based on Fractional Calculus and Natural Selection", Expert Systems with Application Publisher, 39 (2012) 12407-12417.

[21] Chun-Feng Lu and Chia-Feng Juang, Evolutionary fuzzy control of flexible AC transmission system, IEE Proc.Gener. Transm. Distrib., Vol. 152, No. 4, July 2005.

[22] P. Ghamisi, J. A. Benediktsson, Feature Selection Based on Hybridization of Genetic Algorithm and Particle Swarm Optimization, IEEE Geoscience and Remote Sensing Letters, vol.12, no.2, pp.309-313, Feb. 2015.

[23] P. Ghamisi, "A Novel Method for Segmentation of Remote Sensing Images based on Hybrid GA-PSO", International Journal of Computer Applications, 29(2):7-14, September 2011. Published by Foundation of Computer Science, New York, USA.

[24] P. Ghamisi, F. Sepehrband, L. Kumar, M. S. Couceiro, Fernando M. L. Martins, A New Method for Compression of Remote Sensing Images Based on Enhanced Differential Pulse Code Modulation Transformation, Science Asia, 39 (5), 449-455. 